\title{
$\ell$ GOES TO PLUS INFINITY : AN UPDATE
}

\author{
MICHEL CHIPOT \\ INSTITUTE OF MATHEMATICS, UNIVERSity OF ZURICH, WinTERTHURERSTRASSE 190, CH-8057 ZURICH, \\ SWITZERLAND \\ E-mail address: m.m.chipot@math.uzh.ch
}

\begin{abstract}
The goal of this note is to describe the asymptotic behaviour of problems set in cylinders when the size of them is becoming infinite. This leads to consider problems in unbounded domains as well as new singular perturbations issues.
\end{abstract}

\section{INTRODUCTION}

It has been observed since a long time that the solution of problems set in cylinders, when the data are depending on the section only, are also almost independent of the axial direction - at least, as we will see, on a large portion of the cylinder at stake. At a time when one was trying to save some computation power this has lead many numerical analysts to be convinced that it was enough to carry out the simulations in two dimensions i.e. on the section of the cylinder. If this seems reasonable, trying to justify that mathematically turns out to be often a challenging task which has been the topic of recent research with sometimes unexpected developments in different fields like for instance singular perturbations. Since the publication of the book " $\ell$ goes to plus infinity", which attacks some of the issues mentioned above (Cf. [7], [18]), a lot of progresses have been made in different directions. This is the goal of this note to advertise them and to report on the issues which are still open. We refer to [33], [34] for classical results and notation.

The simplest problem of the type " $\ell$ goes to plus infinity" is the following. Let $\Omega_{\ell}$ be the rectangle defined as

$$
\Omega_{\ell}=(-\ell, \ell) \times(-1,1) .
$$

If we denote by $\left(x_{1}, x_{2}\right)$ the points in $\mathbb{R}^{2}$, let

$$
f=f\left(x_{2}\right) \in L^{2}(-1,1) .
$$

Received by the editors April 21 2014; Accepted May 17 2014; Published online May 252014.

2000 Mathematics Subject Classification. 35J25, 35K20, 46N99.

Key words and phrases. Asymptotic behaviour, Cylinder, Elliptic, Parabolic problems, Periodic data. 


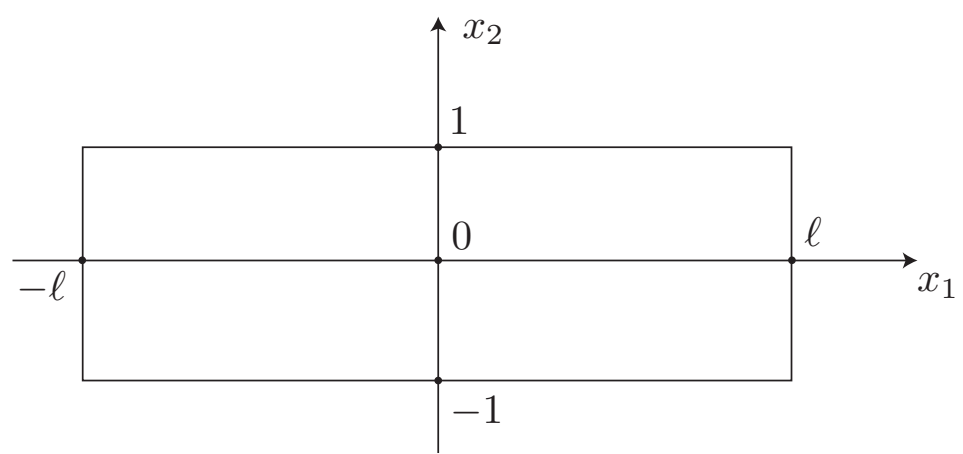

FIGURE 1.1

Then by the Lax-Milgram theorem, it is well known that there exists a unique weak solution to the problem

$$
\begin{cases}-\Delta u_{\ell}=f & \text { in } \Omega_{\ell}, \\ u_{\ell}=0 & \text { on } \partial \Omega_{\ell} .\end{cases}
$$

( $\Delta$ is the usual Laplace operator, $\partial \Omega_{\ell}$ denotes the boundary of $\Omega_{\ell}$ ). The issue is then to determine what happens for $u_{\ell}$ when $\ell$ goes to plus $+\infty$. A natural candidate for the limit is $u_{\infty}$ the solution of a similar problem on the section of the rectangle that is to say the solution to

$$
\left\{\begin{array}{l}
-\partial_{x_{2}}^{2} u_{\infty}=f \quad \text { in }(-1,1), \\
u_{\infty}( \pm 1)=0
\end{array}\right.
$$

One can indeed show (see [29], [8], [47]):

Theorem 1.1. There exists positive constants $C, \alpha$ such that

$$
\|\left.\nabla\left(u_{\ell}-u_{\infty}\right)\right|_{2, \Omega_{\frac{\ell}{2}}} \leq C e^{-\alpha \ell}
$$

( | $\left.\right|_{2, A}$ denotes the usual $L^{2}(A)$-norm, | | the euclidean norm in $\mathbb{R}^{2}$ ).

Remark 1.1. The above theorem states in particular that, on any fixed subdomain of the strip $\Omega_{\infty}=\mathbb{R} \times(-1,1), u_{\ell}$ converges toward $u_{\infty}$ at an exponential rate. Note that $u_{\ell}$ itself is not independent of $x_{1}$ unless it is identically equal to 0 and $f$ as well.

Open question : We do not know if the exponential rate of convergence is preserved when one replaces in (1.3) the Laplace operator by the $p$-Laplace operator. For some convergence results we refer to [24], [28], [45]. 


\section{THE GENERAL FRAMEWORK}

We denote by $\omega_{1}, \omega_{2}$ two bounded open subsets of $\mathbb{R}^{m}$ and $\mathbb{R}^{n-m}$ respectively and we suppose that

$$
0 \in \omega_{1} \text { and } \omega_{1} \text { is star shaped with respect to } 0 \text {. }
$$

For $\ell>0$ we set

$$
\Omega_{\ell}=\ell \omega_{1} \times \omega_{2} .
$$

The points $x=\left(x_{1}, x_{2}, \ldots, x_{n}\right)$ in $\mathbb{R}^{n}$ are split into two components $X_{1}, X_{2}$ where

$$
X_{1}=x_{1}, \ldots, x_{m}, X_{2}=x_{m+1}, \ldots, x_{n}
$$

i.e. $X=\left(X_{1}, X_{2}\right)$.

Let us denote by $f$ a function independent of $X_{1}$, i.e.

$$
f=f\left(X_{2}\right) .
$$

and consider $u_{\ell}$ the weak solution to

$$
\begin{cases}-\partial_{x_{i}}\left(\left|\partial_{x_{i}} u_{\ell}\right|^{p-2} \partial_{x_{i}} u_{\ell}\right)=f & \text { in } \Omega_{\ell} \\ u_{\ell}=0 & \text { on } \partial \Omega_{\ell}\end{cases}
$$

(with the summation convention on $i=1, \ldots, n$ ). When $p=2$ the above operator is the Laplace operator. We will assume in what follows that

$$
p \geq 2 .
$$

Then, if for instance

$$
f \in L^{q}\left(\omega_{2}\right), \frac{1}{p}+\frac{1}{q}=1,
$$

the problem above admits a unique weak solution in $W_{0}^{1, p}\left(\Omega_{\ell}\right)$-i.e. there exists a unique $u_{\ell}$ satisfying (see [8])

$$
\left\{\begin{array}{l}
u_{\ell} \in W_{0}^{1, p}\left(\Omega_{\ell}\right), \\
\int_{\Omega_{\ell}}\left|\partial_{x_{i}} u_{\ell}\right|^{p-2} \partial_{x_{i}} u_{\ell} \partial_{x_{i}} v d x=\int_{\Omega_{\ell}} f v d x \forall v \in W_{0}^{1, p}\left(\Omega_{\ell}\right) .
\end{array}\right.
$$

We would like to find out the limit behaviour of $u_{\ell}$ when $\ell \rightarrow+\infty$. For that we will assume that $\omega_{1}$ - in addition to (2.1) - possesses the following property. For every $a>0$ there exists a function $\rho_{a}=\rho_{a}\left(X_{1}\right)$ such that

$$
0 \leq \rho_{a} \leq 1, \rho_{a}=1 \text { on } a \omega_{1}, \rho_{a}=0 \text { on } \partial\left\{(a+1) \omega_{1}\right\},\left|\nabla \rho_{a}\right| \leq C
$$

for some constant $C$.

Remark 2.1. If $\omega_{1}$ is convex the property above holds true. 
Then, if $u_{\infty}$ denotes the solution to

$$
\left\{\begin{array}{l}
u_{\infty} \in W_{0}^{1, p}\left(\omega_{2}\right), \\
\int_{\omega_{2}}\left|\partial_{x_{i}} u_{\infty}\right|^{p-2} \partial_{x_{i}} u_{\infty} \partial_{x_{i}} v d X_{2}=\int_{\omega_{2}} f v d X_{2} \forall v \in W_{0}^{1, p}\left(\omega_{2}\right),
\end{array}\right.
$$

(note that in the integral above the summation convention is made for $i=m+1, \ldots, n, d X_{2}=$ $\left.d x_{m+1} \ldots d x_{n}\right)$, we have :

Theorem 2.1. There exists positive constants $C, \alpha$ such that

$$
\|\left.\nabla\left(u_{\ell}-u_{\infty}\right)\right|_{p, \Omega_{\frac{\ell}{2}}} \leq C e^{-\alpha \ell}
$$

Proof. Let us denote by $\ell_{1}$ a real number such that $0<\ell_{1} \leq \ell-1$. It is clear that

$$
\rho_{\ell_{1}}\left(X_{1}\right)\left(u_{\ell}-u_{\infty}\right) \in W_{0}^{1, p}\left(\Omega_{\ell}\right)
$$

and thus it is a suitable test function for (2.8). Moreover for a.e. $X_{1}$ (Cf. [7])

$$
\rho_{\ell_{1}}\left(X_{1}\right)\left(u_{\ell}-u_{\infty}\right)\left(X_{1}, .\right) \in W_{0}^{1, p}\left(\omega_{2}\right) .
$$

and thus is a suitable test function for (2.10). Thus, for a.e. $X_{1}$ it holds

$$
\int_{\omega_{2}}\left|\partial_{x_{i}} u_{\infty}\right|^{p-2} \partial_{x_{i}} u_{\infty} \partial_{x_{i}}\left\{\rho_{\ell_{1}}\left(u_{\ell}-u_{\infty}\right)\right\} d X_{2}=\int_{\omega_{2}} f\left\{\rho_{\ell_{1}}\left(u_{\ell}-u_{\infty}\right)\right\} d X_{2}
$$

(Note that in the first integral above $i$ can run from 1 to $n$ since $u_{\infty}$ is independent of $X_{1}$ ). Integrating (2.14) in $X_{1}$ we get

$$
\int_{\Omega_{\ell}}\left|\partial_{x_{i}} u_{\infty}\right|^{p-2} \partial_{x_{i}} u_{\infty} \partial_{x_{i}}\left\{\rho_{\ell_{1}}\left(u_{\ell}-u_{\infty}\right)\right\} d x=\int_{\Omega_{\ell}} f\left\{\rho_{\ell_{1}}\left(u_{\ell}-u_{\infty}\right)\right\} d x
$$

Taking $v=\rho_{\ell_{1}}\left(u_{\ell}-u_{\infty}\right)$ in (2.8) and subtracting the equality above we obtain

$$
\int_{\Omega_{\ell}}\left\{\left|\partial_{x_{i}} u_{\ell}\right|^{p-2} \partial_{x_{i}} u_{\ell}-\left|\partial_{x_{i}} u_{\infty}\right|^{p-2} \partial_{x_{i}} u_{\infty}\right\} \partial_{x_{i}}\left\{\rho_{\ell_{1}}\left(u_{\ell}-u_{\infty}\right)\right\} d x=0 .
$$

Since $\rho_{\ell_{1}}$ vanishes outside $\Omega_{\ell_{1}+1}$ it is enough in the integral above to integrate on $\Omega_{\ell_{1}+1}$ and we obtain easily

$$
\begin{aligned}
& \int_{\Omega_{\ell_{1}+1}}\left\{\left|\partial_{x_{i}} u_{\ell}\right|^{p-2} \partial_{x_{i}} u_{\ell}-\left|\partial_{x_{i}} u_{\infty}\right|^{p-2} \partial_{x_{i}} u_{\infty}\right\} \partial_{x_{i}}\left\{u_{\ell}-u_{\infty}\right\} \rho_{\ell_{1}} d x \\
= & \int_{\Omega_{\ell_{1}+1} \backslash \Omega_{\ell_{1}}}\left\{\left|\partial_{x_{i}} u_{\ell}\right|^{p-2} \partial_{x_{i}} u_{\ell}-\left|\partial_{x_{i}} u_{\infty}\right|^{p-2} \partial_{x_{i}} u_{\infty}\right\} \partial_{x_{i}}\left\{\rho_{\ell_{1}}\right\}\left(u_{\ell}-u_{\infty}\right) d x .
\end{aligned}
$$

(We used the fact that $\rho_{\ell_{1}}=1$ on $\Omega_{\ell_{1}}$. In the last integral $i$ runs from 1 to $m$ since $\rho_{\ell_{1}}$ is independent of $X_{2}$ ). It is well known that for some constant $C_{p}$ one has

$$
C_{p}|a-b|^{p} \leq\left(|a|^{p-2} a-|b|^{p-2} b\right)(a-b) \forall a, b \in \mathbb{R}
$$


and thus from (2.17) we derive easily

$$
C_{p} \int_{\Omega_{\ell_{1}+1}}\left|\partial_{x_{i}}\left(u_{\ell}-u_{\infty}\right)\right|^{p} \rho_{\ell_{1}} d x \leq \int_{\Omega_{\ell_{1}+1} \backslash \Omega_{\ell_{1}}}\left|\partial_{x_{i}} u_{\ell}\right|^{p-1}\left|\partial_{x_{i}} \rho_{\ell_{1}}\right|\left|u_{\ell}-u_{\infty}\right| d x .
$$

Since $\rho_{\ell_{1}}=1$ on $\Omega_{\ell_{1}}$ and $u_{\infty}$ is independent of $X_{1}$ it implies that

$$
C_{p} \int_{\Omega_{\ell_{1}}}\left|\partial_{x_{i}}\left(u_{\ell}-u_{\infty}\right)\right|^{p} d x \leq \int_{\Omega_{\ell_{1}+1} \backslash \Omega_{\ell_{1}}}\left|\partial_{x_{i}}\left(u_{\ell}-u_{\infty}\right)\right|^{p-1}\left|\partial_{x_{i}} \rho_{\ell_{1}}\right|\left|u_{\ell}-u_{\infty}\right| d x .
$$

(Note that in the last integral $i$ is running from 1 to $m$ ). Using the equivalence of norms in $\mathbb{R}^{n}$ and (2.9) we derive easily

$$
\int_{\Omega_{\ell_{1}}}\left|\nabla\left(u_{\ell}-u_{\infty}\right)\right|^{p} d x \leq C \int_{\Omega_{\ell_{1}+1} \backslash \Omega_{\ell_{1}}}\left|\nabla_{X_{1}}\left(u_{\ell}-u_{\infty}\right)\right|^{p-1}\left|u_{\ell}-u_{\infty}\right| d x
$$

where $C=C(p, m, n)$ is a constant depending on $p, m$ and $n,|\quad|$ is the euclidean norm, $\nabla_{X_{1}}=\left(\partial_{x_{1}}, \ldots, \partial_{x_{m}}\right)$ is the gradient in $X_{1}$. Using then the Young inequality

$$
a b \leq \frac{a^{q}}{q}+\frac{b^{p}}{p}
$$

with $q=\frac{p}{p-1}$ we arrive to

$$
\int_{\Omega_{\ell_{1}}}\left|\nabla\left(u_{\ell}-u_{\infty}\right)\right|^{p} d x \leq C \int_{\Omega_{\ell_{1}+1} \backslash \Omega_{\ell_{1}}}\left|\nabla_{X_{1}}\left(u_{\ell}-u_{\infty}\right)\right|^{p}+\left|u_{\ell}-u_{\infty}\right|^{p} d x .
$$

By the Poincaré inequality we have for a.e. $X_{1} \in \ell \omega_{1}$

$$
\int_{\omega_{2}}\left|\left(u_{\ell}-u_{\infty}\right)\right|^{p}\left(X_{1}, X_{2}\right) d X_{2} \leq C\left(\omega_{2}\right) \int_{\omega_{2}}\left|\nabla_{X_{2}}\left(u_{\ell}-u_{\infty}\right)\right|^{p}\left(X_{1}, X_{2}\right) d X_{2}
$$

for some constant $C\left(\omega_{2}\right), \nabla_{X_{2}}=\left(\partial_{x_{m+1}}, \ldots, \partial_{x_{n}}\right)$ is the gradient in $X_{2}$. Integrating in $X_{1}$ we derive

$$
\int_{\Omega_{\ell_{1}+1} \backslash \Omega_{\ell_{1}}}\left|\left(u_{\ell}-u_{\infty}\right)\right|^{p} d x \leq C\left(\omega_{2}\right) \int_{\Omega_{\ell_{1}+1} \backslash \Omega_{\ell_{1}}}\left|\nabla_{X_{2}}\left(u_{\ell}-u_{\infty}\right)\right|^{p} d x .
$$

Thus going back to (2.22) we get for some constant $C$

$$
\int_{\Omega_{\ell_{1}}}\left|\nabla\left(u_{\ell}-u_{\infty}\right)\right|^{p} d x \leq C \int_{\Omega_{\ell_{1}+1} \backslash \Omega_{\ell_{1}}}\left|\nabla\left(u_{\ell}-u_{\infty}\right)\right|^{p} d x
$$

which leads to

$$
\int_{\Omega_{\ell_{1}}}\left|\nabla\left(u_{\ell}-u_{\infty}\right)\right|^{p} d x \leq \frac{C}{C+1} \int_{\Omega_{\ell_{1}+1}}\left|\nabla\left(u_{\ell}-u_{\infty}\right)\right|^{p} d x .
$$

Starting from $\ell_{1}=\frac{\ell}{2}$ and iterating this inequality $\left[\frac{\ell}{2}\right]$-times where [ ] denotes the integer part of a real we get

$$
\int_{\Omega_{\frac{\ell}{2}}}\left|\nabla\left(u_{\ell}-u_{\infty}\right)\right|^{p} d x \leq\left(\frac{C}{C+1}\right)^{\left[\frac{\ell}{2}\right]} \int_{\Omega_{\frac{\ell}{2}+\left[\frac{\ell}{2}\right]}}\left|\nabla\left(u_{\ell}-u_{\infty}\right)\right|^{p} d x .
$$


Setting $\gamma=\frac{C}{C+1}<1$ and noticing that

$$
\frac{\ell}{2}-1<\left[\frac{\ell}{2}\right] \leq \frac{\ell}{2}
$$

we obtain

$$
\int_{\Omega_{\frac{\ell}{2}}}\left|\nabla\left(u_{\ell}-u_{\infty}\right)\right|^{p} d x \leq \gamma^{\frac{\ell}{2}-1} \int_{\Omega_{\ell}}\left|\nabla\left(u_{\ell}-u_{\infty}\right)\right|^{p} d x .
$$

We try now to evaluate the right hand side integral above. Taking $v=u_{\ell}$ in (2.8) we get easily using in particular the Poincaré inequality :

$$
\begin{aligned}
\int_{\Omega_{\ell}}\left|\partial_{x_{i}} u_{\ell}\right|^{p} d x & =\int_{\Omega_{\ell}} f u_{\ell} d x \\
& \leq|f|_{q, \Omega_{\ell}}\left|u_{\ell}\right|_{p, \Omega_{\ell}} \\
& \leq|f|_{q, \Omega_{\ell}} C\left(\omega_{2}\right)|| \nabla_{X_{2}} u_{\ell}||_{p, \Omega_{\ell}} \\
& \leq C\left(\omega_{2}\right)|f|_{q, \Omega_{\ell}}|| \nabla u_{\ell}||_{p, \Omega_{\ell}}
\end{aligned}
$$

and thus, by equivalence of the norms in $\mathbb{R}^{n}$

$$
\begin{aligned}
|| \nabla u_{\ell}||_{p, \Omega_{\ell}}^{p} & \leq C|f|_{q, \Omega_{\ell}}|| \nabla u_{\ell}||_{p, \Omega_{\ell}} \\
\Rightarrow|| \nabla u_{\ell}||_{p, \Omega_{\ell}}^{p} & \leq C|f|_{q, \Omega_{\ell}}^{\frac{p}{p-1}}=C\left(\int_{\ell \omega_{1}} \int_{\omega_{2}}\left|f\left(X_{2}\right)\right|^{q} d x\right) \\
& \leq C \ell^{m}|f|_{q, \omega_{2}}^{\frac{p}{p-1}}
\end{aligned}
$$

where $C=C\left(\omega_{1}, \omega_{2}, m, n\right)$. Similarly taking $v=u_{\infty}$ in (2.10) we derive

$$
\left.|| \nabla u_{\infty}\right|_{p, \omega_{2}} ^{p} \leq C|f|_{q, \omega_{2}}^{\frac{p}{p-1}}
$$

and thus

$$
\left.|| \nabla u_{\infty}\right|_{p, \Omega_{\ell}} ^{p} \leq C \ell^{m}|f|_{q, \omega_{2}}^{\frac{p}{p-1}}
$$

Going back to (2.29) we get

$$
\begin{aligned}
\left\{\int_{\Omega_{\ell}}\left|\nabla\left(u_{\ell}-u_{\infty}\right)\right|^{p} d x\right\}^{\frac{1}{p}} & \leq\left(\frac{e^{\frac{\ell}{2} \ln \gamma}}{\gamma}\right)^{\frac{1}{p}}\left\{\int_{\Omega_{\ell}}\left|\nabla\left(u_{\ell}-u_{\infty}\right)\right|^{p} d x\right\}^{\frac{1}{p}} \\
& \leq C|f|_{q, \omega_{\ell}}^{\frac{1}{p-1}} \ell^{\frac{m}{p}} e^{\frac{\ell}{2 p} \ln \gamma} \\
& \leq C e^{-\alpha \ell},
\end{aligned}
$$

for any $\alpha<-\frac{\ln \gamma}{2 p}$. This completes the proof of the theorem. 
Remark 2.2. In the case $p=2$ we have obtained at the same time the proof of Theorem 1.1. Note that in the case of the $p$-Laplace operator i.e. when

$$
-\Delta_{p} u=-\partial_{x_{i}}\left\{|\nabla u|^{p-2} \partial_{x_{i}}\right\}
$$

the technique above does not work since all the derivatives are involved in the operator (see (2.20)).

\section{PERIODIC PROBLEMS}

In the two sections above we considered a function $f$ constant in some directions. A natural generalization is to consider a $f$ which is periodic - in one direction or several. For instance if we consider (1.3) with $f$ periodic in $x_{1}$ does it force $u_{\ell}$ to converge toward a periodic function? The answer is yes and we refer the reader to [8] for a proof and some generalizations. What we would like to do in this section is to prove that this works also in a parabolic framework. We consider again the simple case of $\Omega_{\ell}$ given by (1.1) and a function

$$
f=f\left(x_{1}, x_{2}\right)
$$

periodic in $x_{1}$ with period $P$-i.e. we assume

$$
f\left(x_{1}+P, x_{2}\right)=f\left(x_{1}, x_{2}\right) \text { a.e. } x .
$$

We denote by $C$ the period cell

$$
C=(0, P) \times(-1,1)
$$

where it is enough to define $f$ and we assume that

$$
f \in L^{2}(C) .
$$

We define

$$
\begin{gathered}
C_{0, p e r}^{1}(C)=\left\{v \in C^{1}(\bar{C}): v(., \pm 1)=0, v(0, .)=v(P, .)\right\} \\
V=H_{0, p e r}^{1}(C)=\text { the closure of } C_{0, p e r}^{1}(C) \text { in } H^{1}(C)
\end{gathered}
$$

i.e. $V=H_{0, p e r}^{1}(C)$ is the set of $H^{1}(C)$-functions periodic in $x_{1}$ and vanishing on the part of the boundary of $C$ contained in the straight lines $x_{2}= \pm 1$. It is clear that $H_{0, p e r}^{1}(C)$ is a Hilbert space when equipped with the Dirichlet norm

$$
\left(\int_{C}|\nabla u|^{2} d x\right)^{\frac{1}{2}}
$$


Clearly $f \in V^{\prime}$ the dual of $V$ and for $u^{0} \in L^{2}(C)$ there exists a unique $u_{\infty}$ (this notation will be clear later) solution to

$$
\left\{\begin{array}{l}
u_{\infty} \in L^{2}(0, T ; V), \partial_{t} u_{\infty} \in L^{2}\left(0, T ; V^{\prime}\right) \\
\partial_{t}\left(u_{\infty}, v\right)+\int_{C} \nabla u_{\infty} \cdot \nabla v d x=\int_{C} f v d x \forall v \in V \text { in } \mathcal{D}^{\prime}(0, T), \\
u_{\infty}(0, .)=u^{0}
\end{array}\right.
$$

( $T$ is a positive number). We suppose from now on that $u_{\infty}$ and $u^{0}$ are extended by periodicity $P$ on the whole strip $\mathbb{R} \times(-1,1)$. For $\ell>0$ we set

$$
\Omega_{\ell}=(-\ell P, \ell P) \times(-1,1), V_{\ell}=H_{0}^{1}\left(\Omega_{\ell}\right) .
$$

We suppose $V_{\ell}$ equipped with the Dirichlet norm - i.e. defined by (3.1) with $C$ replaced by $\Omega_{\ell}$. Then $f \in V_{\ell}^{\prime}$ and there exists a unique $u_{\ell}$ solution to

$$
\left\{\begin{array}{l}
u_{\ell} \in L^{2}\left(0, T ; V_{\ell}\right), \partial_{t} u_{\ell} \in L^{2}\left(0, T ; V_{\ell}^{\prime}\right), \\
\partial_{t}\left(u_{\ell}, v\right)+\int_{\Omega_{\ell}} \nabla u_{\ell} \cdot \nabla v d x=\int_{\Omega_{\ell}} f v d x \forall v \in V_{\ell} \text { in } \mathcal{D}^{\prime}(0, T), \\
u_{\ell}(0, .)=u^{0}
\end{array}\right.
$$

Then we have :

Theorem 3.1. When $\ell \rightarrow+\infty, u_{\ell} \rightarrow u_{\infty}$ with an exponential rate of convergence. (i.e. the periodicity of $f$ forces $u_{\ell}$ to be periodic at the limit).

We will need the following lemma:

Lemma 3.1. Let $u_{\infty}$ be the periodic extension of the solution to (3.2). Then for every $\Omega \subset$ $\mathbb{R} \times(-1,1)$ bounded, it holds

$$
\partial_{t}\left(u_{\infty}, v\right)+\int_{\Omega} \nabla u_{\infty} \cdot \nabla v d x=\int_{\Omega} f v d x \forall v \in H_{0}^{1}(\Omega) \text { in } \mathcal{D}^{\prime}(0, T) .
$$

Proof. For $z \in \mathbb{Z}$ we denote by $C_{z}$ the translated of $C=C_{0}$ the reference cell i.e.

$$
C_{z}=C_{0}+(z P, 0) \text {. }
$$

We suppose that $v$ is extended by 0 outside $\Omega$. Then the support of $v$ intersects only a finite number of cells $C_{z}$-see the figure below.

In $\mathcal{D}^{\prime}(0, T)$ one considers the distribution

$$
A=\partial_{t}\left(u_{\infty}, v\right)+\int_{\Omega} \nabla u_{\infty} \cdot \nabla v d x=\partial_{t}\left(\sum_{z}\left(u_{\infty}, v\right)_{2, C_{z}}\right)+\sum_{z} \int_{C_{z}} \nabla u_{\infty} \cdot \nabla v d x
$$




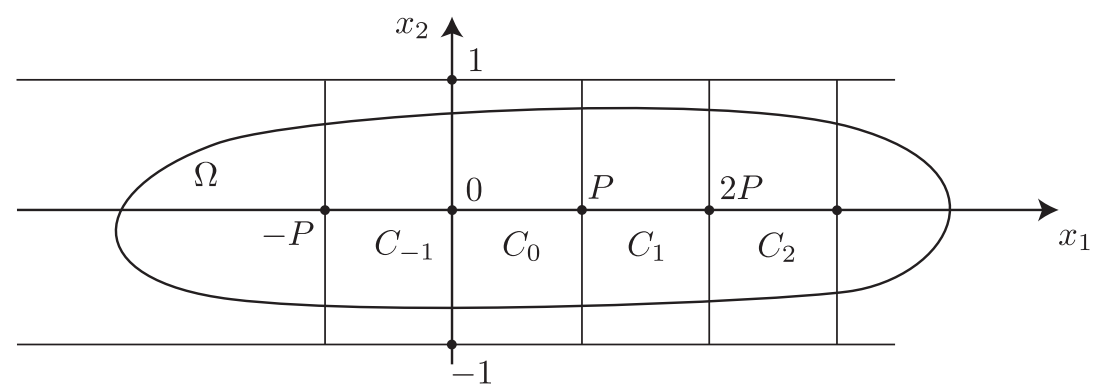

FIGURE 3.2

(( , $)_{2, D}$ denotes the usual $L^{2}(D)$-scalar product). Since $C_{z}=C_{0}+(z P, 0)$ one has

$$
\begin{aligned}
A= & \partial_{t}\left\{\sum_{z}\left(u_{\infty}\left(t, x_{1}+z P, x_{2}\right), v\left(x_{1}+z P, x_{2}\right)\right)_{2, C}\right\} \\
& +\sum_{z} \int_{C} \nabla u_{\infty}\left(t, x_{1}+z P, x_{2}\right) \cdot \nabla v\left(x_{1}+z P, x_{2}\right) d x .
\end{aligned}
$$

Since $u_{\infty}$ has no jump through the different cells, $u_{\infty}$ and $\nabla u_{\infty}$ are periodic of period $P$ in the $x_{1}$ direction and thus

$$
A=\partial_{t}\left(u_{\infty}, \sum_{z} v\left(x_{1}+z P, x_{2}\right)\right)_{2, C}+\int_{C} \nabla u_{\infty} \cdot \nabla\left\{\sum_{z} v\left(x_{1}+z P, x_{2}\right)\right\} d x .
$$

It is clear that the function

$$
\sum_{z} v\left(x_{1}+z P, x_{2}\right)
$$

is $P$-periodic in $x_{1}$ and vanishes on $x_{2}= \pm 1$ (note that only a finite number of terms in the sum are different of 0$)$. Thus by (3.2) we have in $\mathcal{D}^{\prime}(0, T)$

$$
\begin{aligned}
A & =\int_{C} f\left(x_{1}, x_{2}\right) \sum_{z} v\left(x_{1}+z P, x_{2}\right) d x \\
& =\sum_{z} \int_{C} f\left(x_{1}+z P, x_{2}\right) v\left(x_{1}+z P, x_{2}\right) d x=\sum_{z} \int_{C_{z}} f v d x=\int_{\Omega} f v d x
\end{aligned}
$$

since $f$ is periodic in the direction $x_{1}$. This completes the proof of the Lemma.

Proof of the Theorem For $\ell_{1} \leq \ell-1$ we denote by $\rho=\rho_{\ell_{1}}$ the function which graph is described below (compare to (2.9)).

Clearly one has

$$
\left(u_{\ell}-u_{\infty}\right) \rho\left(x_{1}\right) \in V_{\ell}
$$




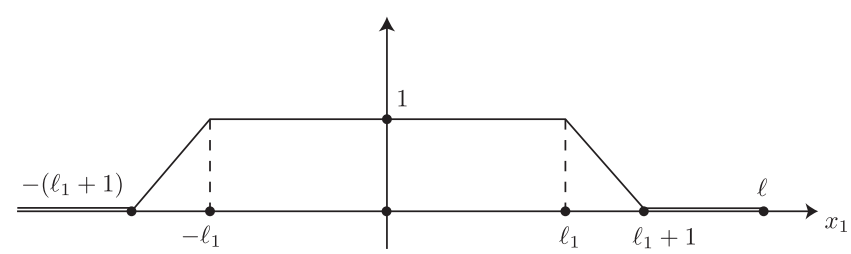

FIGURE 3.3

and from (3.3), (3.4) one deduces (see [6] for the notation $<,>$ )

$$
<\partial_{t}\left(u_{\ell}-u_{\infty}\right),\left(u_{\ell}-u_{\infty}\right) \rho>+\int_{\Omega_{\ell_{1}+1}} \nabla\left(u_{\ell}-u_{\infty}\right) \cdot \nabla\left\{\left(u_{\ell}-u_{\infty}\right) \rho\right\} d x=0
$$

i.e.

$$
\begin{aligned}
\frac{1}{2} \frac{d}{d t} \int_{\Omega_{\ell_{1}+1}}\left(u_{\ell}-u_{\infty}\right)^{2} \rho d x & +\int_{\Omega_{\ell_{1}+1}}\left|\nabla\left(u_{\ell}-u_{\infty}\right)\right|^{2} \rho d x \\
& =\int_{D_{\ell_{1}}} \partial_{x_{1}}\left(u_{\ell}-u_{\infty}\right)\left(\partial_{x_{1}} \rho\right)\left(u_{\ell}-u_{\infty}\right) d x
\end{aligned}
$$

where we have set

$$
D_{\ell_{1}}=\Omega_{\ell_{1}+1} \backslash \Omega_{\ell_{1}} .
$$

Since $\left|\partial_{x_{1}} \rho\right| \leq 1$ we derive by Young's inequality

$$
\begin{aligned}
\frac{1}{2} \frac{d}{d t} \int_{\Omega_{\ell_{1}+1}}\left(u_{\ell}-u_{\infty}\right)^{2} \rho d x & +\int_{\Omega_{\ell_{1}}}\left|\nabla\left(u_{\ell}-u_{\infty}\right)\right|^{2} d x \\
\leq & \frac{1}{2} \int_{D_{\ell_{1}}} \partial_{x_{1}}\left(u_{\ell}-u_{\infty}\right)^{2}+\left(u_{\ell}-u_{\infty}\right)^{2} d x .
\end{aligned}
$$

By the Poincaré inequality one has

$$
\int_{D_{\ell_{1}}}\left(u_{\ell}-u_{\infty}\right)^{2} d x \leq 2 \int_{D_{\ell_{1}}} \partial_{x_{2}}\left(u_{\ell}-u_{\infty}\right)^{2}
$$

and thus it holds

$$
\frac{1}{2} \frac{d}{d t} \int_{\Omega_{\ell_{1}+1}}\left(u_{\ell}-u_{\infty}\right)^{2} \rho d x+\int_{\Omega_{\ell_{1}}}\left|\nabla\left(u_{\ell}-u_{\infty}\right)\right|^{2} d x \leq \int_{D_{\ell_{1}}}\left|\nabla\left(u_{\ell}-u_{\infty}\right)\right|^{2} d x .
$$

This can be written as

$$
\frac{1}{2} \frac{d}{d t} \int_{\Omega_{\ell_{1}+1}}\left(u_{\ell}-u_{\infty}\right)^{2} \rho d x+2 \int_{\Omega_{\ell_{1}}}\left|\nabla\left(u_{\ell}-u_{\infty}\right)\right|^{2} d x \leq \int_{\Omega_{\ell_{1}+1}}\left|\nabla\left(u_{\ell}-u_{\infty}\right)\right|^{2} d x .
$$

Integrating between 0 and $T$ we obtain (recall that $u_{\ell}=u_{\infty}=u^{0}$ for $t=0$ ) 


$$
\frac{1}{2} \int_{\Omega_{\ell_{1}}}\left(u_{\ell}-u_{\infty}\right)^{2}(T, x) d x+2 \int_{0}^{T} \int_{\Omega_{\ell_{1}}}\left|\nabla\left(u_{\ell}-u_{\infty}\right)\right|^{2} d x \leq \int_{0}^{T} \int_{\Omega_{\ell_{1}+1}}\left|\nabla\left(u_{\ell}-u_{\infty}\right)\right|^{2} d x
$$

which implies

$$
\begin{aligned}
& \frac{1}{2} \int_{\Omega_{\ell_{1}}}\left(u_{\ell}-u_{\infty}\right)^{2}(T, x) d x+2 \int_{0}^{T} \int_{\Omega_{\ell_{1}}}\left|\nabla\left(u_{\ell}-u_{\infty}\right)\right|^{2} d x \\
& \quad \leq \frac{1}{2}\left\{\frac{1}{2} \int_{\Omega_{\ell_{1}+1}}\left(u_{\ell}-u_{\infty}\right)^{2}(T, x) d x+2 \int_{0}^{T} \int_{\Omega_{\ell_{1}+1}}\left|\nabla\left(u_{\ell}-u_{\infty}\right)\right|^{2} d x\right\} .
\end{aligned}
$$

Setting

$$
F\left(\ell_{1}\right)=\frac{1}{2} \int_{\Omega_{\ell_{1}}}\left(u_{\ell}-u_{\infty}\right)^{2}(T, x) d x+2 \int_{0}^{T} \int_{\Omega_{\ell_{1}}}\left|\nabla\left(u_{\ell}-u_{\infty}\right)\right|^{2} d x d t
$$

we have obtained

$$
F\left(\ell_{1}\right) \leq \frac{1}{2} F\left(\ell_{1}+1\right), \forall \ell_{1} \leq \ell-1 .
$$

Starting from $\ell_{1}=\frac{\ell}{2}$ and iterating this inequality $\left[\frac{\ell}{2}\right]$-times one derives

$$
F\left(\frac{\ell}{2}\right) \leq\left(\frac{1}{2}\right)^{\left[\frac{\ell}{2}\right]} F\left(\frac{\ell}{2}+\left[\frac{\ell}{2}\right]\right) .
$$

Using again (2.28) we get

$$
F\left(\frac{\ell}{2}\right) \leq 2\left(\frac{1}{2}\right)^{\frac{\ell}{2}} F(\ell)=2 e^{-\frac{\ell}{2} \ln 2} F(\ell) .
$$

In order to conclude we need to estimate $F(\ell)$. Taking $v=u_{\ell}$ in (3.3) and integrating in $t$ we get

$$
\begin{aligned}
\frac{1}{2}\left|u_{\ell}\right|_{2, \Omega_{\ell}}^{2}(T)- & \frac{1}{2}\left|u^{0}\right|_{2, \Omega_{\ell}}^{2}+\int_{0}^{T} \int_{\Omega_{\ell}}\left|\nabla u_{\ell}\right|^{2} d x d t=\int_{0}^{T} \int_{\Omega_{\ell}} f u_{\ell} d x d t \\
\leq & \int_{0}^{T}|f|_{2, \Omega_{\ell}}^{2} d t+\frac{1}{4} \int_{0}^{T}\left|u_{\ell}\right|_{2, \Omega_{\ell}}^{2} \\
& \leq \int_{0}^{T}|f|_{2, \Omega_{\ell}}^{2} d t+\frac{1}{2} \int_{0}^{T} \int_{\Omega_{\ell}}\left|\nabla u_{\ell}\right|^{2} d x d t .
\end{aligned}
$$

This leads to

$$
\frac{1}{2}\left|u_{\ell}\right|_{2, \Omega_{\ell}}^{2}(T)+\frac{1}{2} \int_{0}^{T} \int_{\Omega_{\ell}}\left|\nabla u_{\ell}\right|^{2} d x d t \leq \frac{1}{2}\left|u^{0}\right|_{2, \Omega_{\ell}}^{2}+\int_{0}^{T} \int_{\Omega_{\ell}} f^{2} d x d t \leq C\left(u^{0}, f, T\right) \ell .
$$

The same estimate holds for $u_{\infty}$ so that

$$
F(\ell) \leq C \ell
$$


for some constant independent of $\ell$. It follows that

$$
\int_{\Omega_{\frac{\ell}{2}}}\left(u_{\ell}-u_{\infty}\right)^{2}(T, x) d x+\int_{0}^{T} \int_{\Omega_{\frac{\ell}{2}}}\left|\nabla\left(u_{\ell}-u_{\infty}\right)\right|^{2} d x d t \leq C \ell e^{-\frac{\ell}{2} \ln 2} \leq C e^{-\alpha \ell}
$$

for every $\alpha<\frac{\ln 2}{2}$. This completes the proof of the theorem.

\section{EXISTENCE RESULTS IN UNBOUNDED DOMAINS}

When considering (1.3) there is no reason to assume $f$ independent of $x_{1}$. Indeed assuming for instance $f \in L^{2}\left(\Omega_{\ell}\right)$ for every $\ell$ then $u_{\ell}$ is perfectly defined. The question is then to find out if $u_{\ell}$ possesses a limit when $\ell \rightarrow+\infty$. Of course a natural candidate is $u_{\infty}$ solution to

$$
\left\{\begin{array}{l}
-\Delta u_{\infty}=f\left(x_{1}, x_{2}\right) \quad \text { in } \Omega_{\infty}=\mathbb{R} \times(-1,1), \\
u_{\infty}(., \pm 1)=0 .
\end{array}\right.
$$

Unfortunately the solution to (4.1), if it exists, is not unique. Indeed, for instance the functions

$$
u_{\infty}=C e^{\frac{\pi}{2} x_{1}} \cos \left(\frac{\pi}{2} x_{2}\right)
$$

all satisfy (4.1) for $f=0$. Now the only bounded function of the type (4.2) is obtained when $C=0$. Besides non uniqueness it is also not clear that existence of a solution to (4.1) does exist. Indeed when

$$
f \in H^{-1}\left(\Omega_{\infty}\right)
$$

then certainly the Lax-Milgram theorem provides existence and uniqueness of a solution in $H_{0}^{1}\left(\Omega_{\infty}\right)$ to (4.1) (this solution being 0 when $f=0$ ). But they are many simple functions which are not in $H^{-1}\left(\Omega_{\infty}\right)$ (see [31]). For instance this is the case for a function $f=f\left(x_{2}\right) \neq 0$. Indeed consider such a function. If $f \in H^{-1}\left(\Omega_{\infty}\right)$ there exists a unique solution to

$$
u \in H_{0}^{1}\left(\Omega_{\infty}\right), \int_{\Omega_{\infty}} \nabla u \cdot \nabla v d x=<f, v>\forall v \in H_{0}^{1}\left(\Omega_{\infty}\right) .
$$

By uniqueness it is easy to show that $u$ is translation invariant in the $x_{1}$ direction. This implies that $u=u\left(x_{2}\right)$. But this function cannot be in $L^{2}\left(\Omega_{\infty}\right)$ unless $u=f=0$. So in order to establish existence of a solution to (4.1) some new arguments have to be developped. The idea is that if $f$ is not growing too fast at infinity $u_{\ell}$ defined by (1.3) is a Cauchy sequence (see also [43]). We will consider the situation described by the figure below -i.e. $\Omega$ is an unbounded domain contained in the strip $\mathbb{R} \times(-a, a), a>0$. We set

$$
\begin{gathered}
\Omega_{\ell}=(-\ell, \ell) \times(-a, a) \cap \Omega, \\
V_{\ell}=\left\{v \in H^{1}\left(\Omega_{\ell}\right): v=0 \text { on } \partial \Omega_{\ell}\right\}
\end{gathered}
$$




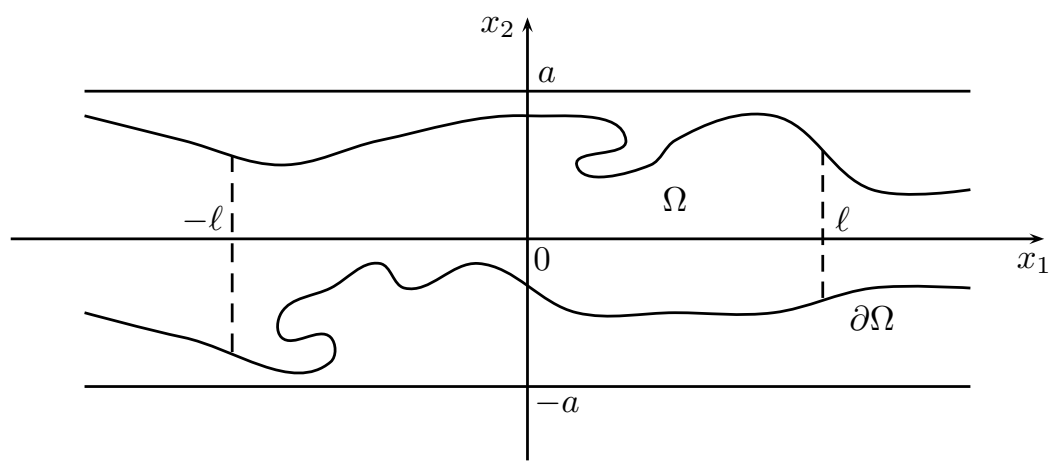

FIGURE 4.4

( $\partial A$ denotes the boundary of $A$ ). Let us denote by $V_{\ell}^{\prime}$ the dual of $V_{\ell}$ and suppose that $f \in V_{\ell}^{\prime}$ for every $\ell>0$. Then there exists a unique $u_{\ell}$ solution to

$$
u_{\ell} \in V_{\ell}, \int_{\Omega_{\ell}} \nabla u_{\ell} \cdot \nabla v d x=<f, v>\forall v \in V_{\ell} .
$$

( $<>$ denotes the $V_{\ell}^{\prime}, V_{\ell}$ duality). Then we have :

Theorem 4.1. Suppose that for some $\gamma>0$ we have

$$
|f|_{V_{\ell}^{\prime}}=O\left(\ell^{\gamma}\right)
$$

where ||$_{V_{\ell}^{\prime}}$ is the strong dual norm in $V_{\ell}^{\prime}$. Then $u_{\ell}$ converges toward the unique solution to

$$
\left\{\begin{array}{l}
u_{\infty} \in H_{l o c}^{1}(\Omega), u_{\infty}=0 \text { on } \partial \Omega, \\
-\Delta u_{\infty}=f \text { in } \Omega,\left\|\nabla u_{\infty}\right\|_{2, \Omega_{\ell}}=O\left(\ell^{\gamma}\right) .
\end{array}\right.
$$

Moreover

$$
\|\left.\nabla\left(u_{\ell}-u_{\infty}\right)\right|_{2, \Omega_{\frac{\ell}{2}}} \leq C e^{-\beta \ell}
$$

for some positive constants $C$ and $\beta$.

Proof. For $\ell_{1} \leq \ell-1$ consider the function $\rho=\rho_{\ell_{1}}$ defined in the preceding section.

a) Estimate of $u_{\ell}-u_{\ell+r}$ for $0 \leq r \leq 1$

One has clearly

$$
\left(u_{\ell}-u_{\ell+r}\right) \rho\left(x_{1}\right) \in V_{\ell} \text { and } V_{\ell+r} .
$$

Thus from (4.3) one deduces

$$
\int_{\Omega_{\ell_{1}+1}} \nabla\left(u_{\ell}-u_{\ell+r}\right) \cdot \nabla\left\{\left(u_{\ell}-u_{\ell+r}\right) \rho\right\} d x=0 .
$$


This implies

$$
\begin{aligned}
\int_{\Omega_{\ell_{1}+1}}\left|\nabla\left(u_{\ell}-u_{\ell+r}\right)\right|^{2} \rho d x & \\
& =\int_{\Omega_{\ell_{1}+1} \backslash \Omega_{\ell_{1}}} \partial_{x_{1}}\left(u_{\ell}-u_{\ell+r}\right) \partial_{x_{1}} \rho\left(u_{\ell}-u_{\ell+r}\right) d x \\
& \leq \int_{D_{\ell_{1}}}\left|\partial_{x_{1}}\left(u_{\ell}-u_{\ell+r}\right)\right|\left|\left(u_{\ell}-u_{\ell+r}\right)\right| d x \\
& \leq \frac{\epsilon}{2} \int_{D_{\ell_{1}}}\left\{\partial_{x_{1}}\left(u_{\ell}-u_{\ell+r}\right)\right\}^{2} d x+\frac{1}{2 \epsilon} \int_{D_{\ell_{1}}}\left(u_{\ell}-u_{\ell+r}\right)^{2} d x
\end{aligned}
$$

( $D_{\ell_{1}}$ has been defined previously, the last inequality follows from Young's inequality). One has the Poincaré inequality (without choosing here the best constant)

$$
\int_{D_{\ell_{1}}}\left(u_{\ell}-u_{\ell+r}\right)^{2} d x \leq 2 a^{2} \int_{D_{\ell_{1}}}\left\{\partial_{x_{2}}\left(u_{\ell}-u_{\ell+r}\right)\right\}^{2} d x
$$

and thus

$$
\int_{\Omega_{\ell_{1}}}\left|\nabla\left(u_{\ell}-u_{\ell+r}\right)\right|^{2} d x \leq \frac{\epsilon}{2} \int_{D_{\ell_{1}}}\left\{\partial_{x_{1}}\left(u_{\ell}-u_{\ell+r}\right)\right\}^{2} d x+\frac{2 a^{2}}{2 \epsilon} \int_{D_{\ell_{1}}}\left\{\partial_{x_{2}}\left(u_{\ell}-u_{\ell+r}\right)\right\}^{2} d x
$$

Choosing $\epsilon=a$ we obtain

$$
\int_{\Omega_{\ell_{1}}}\left|\nabla\left(u_{\ell}-u_{\ell+r}\right)\right|^{2} d x \leq a \int_{\Omega_{\ell_{1}+1} \backslash \Omega_{\ell_{1}}}\left|\nabla\left(u_{\ell}-u_{\ell+r}\right)\right|^{2} d x
$$

i.e.

$$
\int_{\Omega_{\ell_{1}}}\left|\nabla\left(u_{\ell}-u_{\ell+r}\right)\right|^{2} d x \leq \frac{a}{a+1} \int_{\Omega_{\ell_{1}+1}}\left|\nabla\left(u_{\ell}-u_{\ell+r}\right)\right|^{2} d x .
$$

Starting from $\frac{\ell}{2}$ and iterating $\left[\frac{\ell}{2}\right]$ times this inequality we get (see (2.28))

$$
\begin{aligned}
\int_{\Omega_{\frac{\ell}{2}}}\left|\nabla\left(u_{\ell}-u_{\ell+r}\right)\right|^{2} d x & \leq \frac{a+1}{a}\left(\frac{a}{a+1}\right)^{\frac{\ell}{2}} \int_{\Omega_{\ell}}\left|\nabla\left(u_{\ell}-u_{\ell+r}\right)\right|^{2} d x \\
& \leq C e^{-\alpha \ell} \int_{\Omega_{\ell}}\left|\nabla\left(u_{\ell}-u_{\ell+r}\right)\right|^{2} d x
\end{aligned}
$$

with $C=\frac{a+1}{a}, \alpha=-\frac{1}{2} \ln \left(\frac{a+1}{a}\right)$. 


\section{b) Estimate of $u_{\ell}$}

We just take $v=u_{\ell}$ in (4.3) to get

$$
\int_{\Omega_{\ell}}\left|\nabla u_{\ell}\right|^{2} d x=<f, u_{\ell}>\leq|f|_{V_{\ell}^{\prime}}|| \nabla u_{\ell}||_{2, \Omega_{\ell}} .
$$

(We suppose $V_{\ell}$ normed with the Dirichlet norm $|u|_{V_{\ell}}^{2}=\int_{\Omega_{\ell}}|\nabla(u)|^{2} d x$ ). It follows that

$$
\int_{\Omega_{\ell}}\left|\nabla u_{\ell}\right|^{2} d x \leq|f|_{V_{\ell}^{\prime}}^{2}=O\left(\ell^{2 \gamma}\right)
$$

c) $u_{\ell}$ is a Cauchy sequence

Combining (4.8) and (4.9) we have

$$
\begin{aligned}
\int_{\Omega_{\frac{\ell}{2}}}\left|\nabla\left(u_{\ell}-u_{\ell+r}\right)\right|^{2} d x & \leq C e^{-\alpha \ell} 2\left\{\left.|| \nabla u_{\ell}\right|_{2, \Omega_{\ell}} ^{2}+|| \nabla u_{\ell+r} \|_{2, \Omega_{\ell+r}}^{2}\right\} \\
& \leq C^{\prime} e^{-\alpha \ell}\left\{\ell^{2 \gamma}+(\ell+r)^{2 \gamma}\right\} \\
& \leq C^{\prime} \ell^{2 \gamma} e^{-\alpha \ell}\left\{1+\left(1+\frac{r}{\ell}\right)^{2 \gamma}\right\} \leq C^{2} e^{-2 \beta \ell}
\end{aligned}
$$

for some constant $C$ when $\ell>1$-recall that $0 \leq r \leq 1$. This can be written as

$$
\left|u_{\ell}-u_{\ell+r}\right|_{V_{\ell}} \leq C e^{-\beta \ell} \text {. }
$$

Thus for $t$ arbitrary we derive then

$$
\begin{aligned}
\left|u_{\ell}-u_{\ell+t}\right|_{V_{\frac{\ell}{2}}} & \leq\left|u_{\ell}-u_{\ell+1}\right|_{V_{\frac{\ell}{2}}} \\
& +\left|u_{\ell+1}-u_{\ell+2}\right|_{\frac{\ell+1}{2}}+\cdots+\left|u_{\ell+[t]}-u_{\ell+t}\right|_{\frac{\ell+[t]}{2}} \\
& \leq C e^{-\beta \ell}+C e^{-\beta(\ell+1)}+\cdots+C e^{-\beta(\ell+[t])} \\
& \leq C e^{-\beta \ell}\left\{1+e^{-\beta}+\cdots+e^{-\beta[t]}\right\} \leq C \frac{1}{1-e^{-\beta}} e^{-\beta \ell}=C^{\prime} e^{-\beta \ell}
\end{aligned}
$$

independently of $t$.

Suppose that we choose $\ell_{0} \leq \frac{\ell}{2}$ then it follows from above that $u_{\ell}$ is a Cauchy sequence in $H^{1}\left(\Omega_{\ell_{0}}\right)$. It converges toward $u_{\infty}$ such that $u_{\infty}=0$ on $\partial \Omega_{\ell_{0}} \cap \partial \Omega$. Moreover, passing to the limit in $t$ in (4.11) provides (4.6).

\section{d) Limit problem}

From above we have

$$
\int_{\Omega_{\ell_{0}}} \nabla u_{\ell} \cdot \nabla v d x=<f, v>\forall v \in H_{0}^{1}\left(\Omega_{\ell_{0}}\right)
$$


and passing to the limit in $\ell$ we get

$$
\int_{\Omega_{\ell_{0}}} \nabla u_{\infty} \cdot \nabla v d x=<f, v>\forall v \in H_{0}^{1}\left(\Omega_{\ell_{0}}\right) .
$$

e) Estimate of $\left\|\nabla u_{\infty}\right\|_{2, \Omega_{\ell}}$

From (4.11) one has

$$
\left|u_{\ell}-u_{\ell+t}\right|_{V_{\frac{\ell}{2}}} \leq C^{\prime} e^{-\beta \ell}
$$

i.e.

$$
\left|u_{2 \ell}-u_{2 \ell+t}\right|_{V_{\ell}} \leq C^{\prime} e^{-2 \beta \ell}
$$

Passing to the limit in $t$ we obtain

$$
\left|u_{2 \ell}-u_{\infty}\right|_{V_{\ell}} \leq C^{\prime} e^{-2 \beta \ell}
$$

which implies by (4.9)

$$
\begin{aligned}
\left|u_{\infty}\right|_{V_{\ell}} & \leq C^{\prime} e^{-2 \beta \ell}+\left|u_{2 \ell}\right|_{V_{\ell}} \leq C^{\prime} e^{-2 \beta \ell}+\left|u_{2 \ell}\right|_{V_{2 \ell}} \\
& \leq C^{\prime} e^{-\beta \ell}+C^{\prime \prime}(2 \ell)^{\gamma}=O(\ell)^{\gamma} .
\end{aligned}
$$

\section{f) Uniqueness}

Suppose that $u_{\infty}, u_{\infty}^{\prime}$ are two solutions to (4.5). Then one has

$$
\int_{\Omega_{\ell}} \nabla\left(u_{\infty}-u_{\infty}^{\prime}\right) \nabla v d x=0 \forall v \in H_{0}^{1}\left(\Omega_{\ell}\right)
$$

Taking $v=\left(u_{\infty}-u_{\infty}^{\prime}\right) \rho_{\ell_{1}}$ and arguing as above will lead to

$$
\int_{\Omega_{\frac{\ell}{2}}}\left|\nabla\left(u_{\infty}-u_{\infty}^{\prime}\right)\right|^{2} d x \leq C^{2} e^{-2 \beta \ell} .
$$

Letting $\ell \rightarrow \infty$ leads to $u_{\infty}=u_{\infty}^{\prime}$. This completes the proof of the theorem.

Remark 4.1. One can replace the assumption $|f|_{V_{\ell}^{\prime}}=O\left(\ell^{\gamma}\right)$ by $|f|_{V_{\ell}^{\prime}}=O\left(e^{\gamma \ell}\right)$ for some small enough $\gamma$. Note that we are also recovering here the theorem 1.1.

\section{ANISOTROPIC SINGULAR PERTURBATIONS}

Let $u_{\ell}$ the solution to (1.3). Set

$$
v_{\ell}\left(x_{1}, x_{2}\right)=u_{\ell}\left(\ell x_{1}, x_{2}\right) .
$$

Clearly $v_{\ell}$ is defined on $\Omega_{1}=(-1,1)^{2}$. Moreover one has

$$
\partial_{x_{1}}^{2} v_{\ell}\left(x_{1}, x_{2}\right)=\ell^{2} \partial_{x_{1}}^{2} u_{\ell}\left(\ell x_{1}, x_{2}\right), \partial_{x_{2}}^{2} v_{\ell}\left(x_{1}, x_{2}\right)=\partial_{x_{2}}^{2} u_{\ell}\left(\ell x_{1}, x_{2}\right) .
$$


Thus $v_{\ell}$ is solution to

$$
\begin{cases}-\frac{1}{\ell^{2}} \partial_{x_{1}}^{2} v_{\ell}-\partial_{x_{2}}^{2} v_{\ell}=f & \text { in } \Omega_{1} \\ v_{\ell}=0 & \text { on } \partial \Omega_{1}\end{cases}
$$

Setting $\epsilon=\frac{1}{\ell}, v_{\ell}=w_{\epsilon}$ we see that $w_{\epsilon}$ is solution of a problem of the type

$$
\begin{cases}-\epsilon^{2} \partial_{x_{1}}^{2} w_{\epsilon}-\partial_{x_{2}}^{2} w_{\epsilon}=f & \text { in } \Omega_{1}, \\ w_{\epsilon}=0 & \text { on } \partial \Omega_{1} .\end{cases}
$$

This is an anisotropic singular perturbation problem i.e. the diffusion in the direction $x_{1}$ is much smaller when $\epsilon \rightarrow 0$ than in the direction $x_{2}$. The techniques developed for studying $u_{\ell}$ allow to investigate such problems, we refer the interested reader to [9], [10] , [11], [13], [14], [15].

In a more general framework like in our section 2 we have

$$
\Omega_{1}=\omega_{1} \times \omega_{2} .
$$

Consider then a matrix $A$ defined as

$$
A(x)=\left(\begin{array}{ll}
A_{11}(x) & A_{12}\left(X_{2}\right) \\
A_{21}(x) & A_{22}\left(X_{2}\right)
\end{array}\right) .
$$

where $A_{11}$ is a $m \times m$ matrix and $A_{22}$ is a $(n-m) \times(n-m)$ one. Suppose that $A$ is elliptic and in particular satisfies for some constants $\lambda, \Lambda$

$$
\lambda|\xi|^{2} \leq A(x) \xi \cdot \xi,|A(x) \xi| \leq \Lambda|\xi| \forall \xi \in \mathbb{R}^{n} \text {, a.e } x \in \Omega_{1} .
$$

Then a generalization of (5.2) is obtained by setting

$$
A_{\epsilon}(x)=\left(\begin{array}{cc}
\epsilon^{2} A_{11}(x) & \epsilon A_{12}\left(X_{2}\right) \\
\epsilon A_{21}(x) & A_{22}\left(X_{2}\right)
\end{array}\right) .
$$

For $f=f\left(X_{2}\right) \in L^{2}\left(X_{2}\right)$ there exists a unique $w_{\epsilon}$ solution to

$$
\left\{\begin{array}{l}
w_{\epsilon} \in H_{0}^{1}\left(\Omega_{1}\right) \\
\int_{\Omega_{1}} A_{\epsilon}(x) \nabla w_{\epsilon} \cdot \nabla v d x=\int_{\Omega_{1}} f v d x \forall v \in H_{0}^{1}\left(\Omega_{1}\right)
\end{array}\right.
$$

which converges in a certain sense when $\epsilon \rightarrow 0$ toward $w_{0}$ solution to

$$
\left\{\begin{array}{l}
w_{0} \in H_{0}^{1}\left(\omega_{2}\right), \\
\int_{\omega_{2}} A_{22}\left(X_{2}\right) \nabla_{X_{2}} w_{0} \cdot \nabla X_{2} v d X_{2}=\int_{\omega_{2}} f v d X_{2} \forall v \in H_{0}^{1}\left(\omega_{2}\right),
\end{array}\right.
$$

(see [8], [11]).

Remark 5.1. Correctors for such problems can be found in [12]. Also these anisotropic perturbations issues allow to get existence results for exotic problems (see [13], [14]). 


\section{SOME CONCLUDING REMARKS}

We presented above only a few problems which were addressed in the recent years. Many other developments occur. The most important achievement being to reach, with relatively simple techniques, an exponential rate of convergence for the solution of problems set in a domain of the type $\Omega_{\ell}$ toward a solution set in $\Omega_{\infty}$. This gives credit to the numerical analysts' pioneering vision. They indeed did not wait for the theoretical results to reduce in their computations the dimension of the problem.

What can be done for the Dirichlet problem can be extended to the stokes problem in the case of forces independent of one direction as well as in the periodic case (see [1], [16], [17]). It would be interesting to extend this to the two dimensional time dependent Navier-Stokes problem in the spirit of our section 3. Extension in the case of stationary Navier-Stokes problem is more delicate since uniqueness might fail - the same issue occurs for the higher dimensional evolution Navier-Stokes problem. Some higher order problems are investigated in [4], [38]. Hyperbolic issues are considered in [5], [35], [40], [41]. Regarding singular perturbations a self contained study is available in [15] - see also [10], [11], [36], [38], [40].

If $\lambda_{\ell}^{k}$ denotes the $k$-th eigenvalue of the problem

$$
\begin{cases}-\Delta u_{\ell}=\lambda_{\ell}^{k} u_{\ell} & \text { in } \Omega_{\ell}, \\ u_{\ell}=0 & \text { on } \partial \Omega_{\ell},\end{cases}
$$

where $\Omega_{\ell}=\ell \omega_{1} \times \omega_{2}$ it can be shown that $\lambda_{\ell}^{k}$ converges when $\ell \rightarrow+\infty$ towards the first eigenvalue of the problem

$$
\begin{cases}-\Delta_{X_{2}} u_{\infty}=\lambda^{1} u_{\infty} & \text { in } \omega_{2}, \\ u_{\infty}=0 & \text { on } \partial \omega_{2},\end{cases}
$$

( $\Delta_{X_{2}}$ is the usual Laplace operator in $\omega_{2}$ ) more precisely one has

$$
0 \leq \lambda_{\ell}^{k}-\lambda^{1}=O\left(\frac{1}{\ell^{2}}\right)
$$

when $\ell \rightarrow+\infty$. This is easily extended to general elliptic operators (see [22]) and one has also convergence of the eigenfunctions. The passage to the Neumann case could appear straightforward. It is not so, for instance if one considers for $\Omega_{\ell}$ given by (1.1) the first eigenvalue problem.

$$
\left\{\begin{array}{l}
-\nabla \cdot\left(A \nabla u_{\ell}\right)=\lambda_{\ell}^{1} u_{\ell} \text { in } \Omega_{\ell}, \\
u_{\ell}=0 \text { on } x_{2}= \pm 1, A \nabla u_{\ell} \cdot n=0 \text { on } x_{1}= \pm \ell,
\end{array}\right.
$$

$n$ being the outward unit normal to $\Omega_{\ell}$ one does not have

$$
\lambda_{\ell}^{1} \rightarrow \lambda^{1}
$$

as above unless $A$ is a $2 \times 2$ diagonal matrix. A substantial analysis of this problem and its generalisations is done in [23], [44]. 
As we mentioned already in the case of the $p$-Laplace operators, exponential rates of convergence are more complicated to reach for nonlinear problems (Cf. also [42]). It can go through for obstacle problems but remains open in many situation in the framework of variational inequalities (see [30], [32], [47]). Some quasi linear problems are investigated in [7] and also various different norms are considered (i.e. not only the norm of the gradient). To this respect many results can be extended to reach an exponential rate of convergence.

Since everything extends to the periodic case in the spirit of section 3, many issues remain open there (see [1], [25], [26]).

As we might have convinced the reader, any problem set in a cylinder or in a generalised cylinder of the type (2.2) can be analyse the way we did and lead to interesting issues. In the seventies, one of the first application of the obstacle problem and of variational inequalities was the so called Dam Problem. It was set and studied widely in two dimensions and the invoked reason was that one was assuming that the dam at stake was of cylindrical shape and long enough so that the flow in this porous medium was the same in every cross section of the dam. A rigorous proof of this assertion is still pending and challenging. (See [2], [3]) for notions on the problem.

Acknowledgements: The research leading to these results has received funding from Lithuanian-Swiss cooperation programme to reduce economic and social disparities within the enlarged European Union under project agreement No CH-3-SMM-01/0. It was supported also by the Swiss National Science Foundation under the contracts \# 200021-129807/1 and 200021-146620. Finally the paper was completed during a visit at the Newton Institute in Cambridge and at the Department of Computational Science \& Engineering at Yonsei University in Seoul. The nice atmosphere of these institutions is greatly acknowledged.

\section{REFERENCES}

[1] S. Baillet, A. Henrot, T. Takahashi, Convergence results for a semilinear problem and for a Stokes problem in a periodic geometry. Asymptotic Anal. 50, (2006), 325-337.

[2] C. Baiocchi, Su un problema di frontiera libera connesso a questioni di idraulica. Ann. Mat. Pura Appl. (4) 92 (1972), 107-127.

[3] H. Brezis, D. Kinderlehrer and G. Stampacchia, Sur une nouvelle formulation du problème de l'écoulement à travers une digue. C. R. Acad. Sci. Paris Sr. A-B 287 (1978), no. 9, 711-714.

[4] B. Brighi, S. Guesmia, On elliptic boundary value problems of order $2 m$ in cylindrical domain of large size. Adv. Math. Sci. Appl. 18(1), 2008, 237-250.

[5] B. Brighi, S. Guesmia, Asymptotic behavior of solutions of hyperbolic problems on a cylindrical domain. Disc. Cont. Dyn. Syst. Suppl. 2007, 160-169.

[6] M. Chipot, Elements of Nonlinear Analysis. Birkhäuser Advanced Text, 2000.

[7] M. Chipot, $\ell$ goes to plus infinity. Birkhäuser Advanced Text, 2002.

[8] M. Chipot, Elliptic Equations: An Introductory Course. Birkhäuser Advanced Text, 2009.

[9] M. Chipot, On some anisotropic singular perturbation problems. Asymptotic Analysis, 55 (2007), 125-144. 
[10] M. Chipot, Some Remarks on Anisotropic Singular Perturbation Problems. Proceedings of IUTAM Symposium on the relations of shell, plate, beam and 3D models, Tbilisi, Georgia, April 2007, Springer Edt., (2008), 91-100.

[11] M. Chipot, S. Guesmia, On the asymptotic behavior of elliptic, anisotropic singular perturbations problems. Comm. Pure Applied Anal., 8, 1, (2009), 179-194.

[12] M. Chipot, S. Guesmia, Corrector for some asymptotic problems. Proceedings of the Steklov Institue of Mathematics, Vol 270, (2010), 263-277.

[13] M. Chipot, S. Guesmia, On some anisotropic, nonlocal, parabolic singular perturbations problems. Applicable Analysis, Vol. 90, No. 12, (2011), 1775-1789.

[14] M. Chipot, S. Guesmia, On a class of integro-differential problems. Comm. Pure Applied Analysis, 9, 5, (2010), 1249-1262.

[15] M. Chipot, S. Guesmia and A. Sengouga, Singular Perturbations of Some Nonlinear Problems. Journal of Mathematical Sciences, Vol 176, 6, (2011), 828-843.

[16] M. Chipot, S. Mardare, Asymptotic behaviour of the Stokes problem in cylinders becoming unbounded in one direction. J. Math. Pures Appl. 90, (2008), 133-159.

[17] M. Chipot, S. Mardare, On correctors for the Stokes problem in cylinders. Proceedings of the conference on nonlinear phenomena with energy dissipation, Chiba, November 2007, P. Colli and all Edts, Gakuto International Series, Mathematical Sciences and Applications, Vol 29, Gakkotosho, (2008), 37-52.

[18] M. Chipot, A. Rougirel, On the asymptotic behaviour of the solution of elliptic problems in cylindrical domains becoming unbounded. Communications in Contemporary Mathematics, Vol 4, 1, (2002), 15-44.

[19] M. Chipot, A. Rougirel, On the asymptotic behaviour of the solution of parabolic problems in cylindrical domains of large size in some directions. Discrete an Continuous Dynamical Systems, Series B, 1, (2001), 319-338.

[20] M. Chipot, A. Rougirel, Remarks on the asymptotic behaviour of the solution to parabolic problems in domains becoming unbounded. Nonlinear Analysis, Vol 47, (2001), 3-11.

[21] M. Chipot, A. Rougirel, Local stability under changes of boundary conditions at a far away location. Proceedings of the Fourth European Conference on Elliptic and Parabolic Problems, Rolduc-Gaeta 2001, World Scientific, (2002), 52-65.

[22] M. Chipot, A. Rougirel, On the asymptotic behaviour of the eigenmodes for elliptic problems in domains becoming unbounded. Trans. Amer. Math. Soc. 360 (2008), 3579-3602.

[23] M. Chipot, P. Roy and I. Shafrir, Asymptotics of eigenstates of elliptic problems with mixed boundary data on domains tending to infinity. Asymptotic Analysis 85, (2013), 199-227.

[24] M. Chipot, Y. Xie, On the asymptotic behaviour of the p-Laplace equation in cylinders becoming unbounded. Proceedings of International Conference: Nonlinear PDE's and their Applications, N. Kenmochi, M. Ôtani, S. Zheng Edts., Gakkotosho, (2004), 16-27.

[25] M. Chipot, Y. Xie, On the asymptotic behaviour of elliptic problems with periodic data. C. R. Acad. Sci. Paris, Ser. I 339, (2004), 477-482.

[26] M. Chipot, Y. Xie, Elliptic problems with periodic data: an asymptotic analysis. Journ. Math. Pures et Appl., 85 (2006), 345-370.

[27] M. Chipot, Y. Xie, Asymptotic behaviour of nonlinear parabolic problems with periodic data. Progress in PDE and their applications, Vol 63, Nonlinear elliptic and parabolic problems: A special tribute to the work of H. Brezis, (2005), 147-156, Birkhäuser.

[28] M. Chipot, Y. Xie, Some issues on the p-Laplace equation in cylindrical domains. Proceedings of the Steklov Institue of Mathematics, 261, (2008), 287-294. 
[29] M. Chipot, K. Yeressian, Exponential rates of convergence by an iteration technique. C. R. Acad. Sci. Paris, Ser. I 346, (2008), 21-26.

[30] M. Chipot, K. Yeressian, On the asymptotic behavior of variational inequalities set in cylinders. DCDS, Series A, Vol 33, 11 \& 12, (2013), 4875-4890.

[31] M. Chipot, K. Yeressian, On Some Variational Inequalities in Unbounded Domains. Boll. del UMI, (9), V, (2012), 243-262.

[32] M. Chipot, K. Yeressian, Asymptotic behaviour of the solution to variational inequalities with joint constraints on its value and its gradient. Contemporary Mathematics 594, (2013), 137-154.

[33] L.C. Evans, Partial differential equations. Graduate Studies in Mathematics 19, AMS, 1998.

[34] J.-L. Lions, Quelques méthodes de résolution des problèmes aux limites non linéaires. Dunod-GauthierVillars, Paris, 1969.

[35] S. Guesmia, Some results on the asymptotic behavior for hyperbolic problems in cylindrical domains becoming unbounded. J. Math. Anal. Appl. 341(2), 2008, 1190-1212.

[36] S. Guesmia, Asymptotic behavior of elliptic boundary-value problems with some small coefficients. Electron. J. Diff. Equ. 2008 (59), (2008), 1-13.

[37] S. Guesmia, Some convergence results for quasilinear parabolic boundary value problems in cylindrical domain of large size. Nonlinear Anal. 70(9), 2009, 3320-3331.

[38] S. Guesmia, Résultats sur l'analyse asymptotique des équations aux dérivées partielles. Habilitation, Université de Haute Alsace, France (2013).

[39] S. Guesmia, Large time and space size behaviour of the heat equation in non-cylindrical domains. Arch. Math., (2013), 101 (3), 293-299.

[40] S. Guesmia, A. Sengouga, Some singular perturbations results for semilinear hyperbolic problems. Discrete. Cont. Dyn. Syst. Ser. S, 5(3), 2012, 567-580.

[41] S. Guesmia, A. Sengouga, Anisotropic singular perturbations for hyperbolic problems. Appl. Math. Comput. 217 (22), (2011), 8983-8996.

[42] D. Mugnai, A limit problem for degenerate quasilinear variational inequalities in cylinders. Recent trends in nonlinear partial differential equations. I. Evolution problems, Contemp. Math., 594, Amer. Math. Soc., Providence, RI, (2013), 281-293.

[43] O. A. Oleinik and G. A. Yosifian, Boundary value problems for second order elliptic equations in unbounded domains and Saint-Venants principle. Ann. Scuola Norm. Sup. Pisa Cl. Sci. (4), 4, no. 2, (1977), 269-290.

[44] P. Roy, Some results in asymptotic analysis and nonlocal problems. Thesis University of Zürich, (2013).

[45] Y. Xie, On asymptotic problems in Cylinders and other mathematical issues. Thesis University of Zürich, (2006).

[46] K. Yeressian, Asymptotic Behavior of Elliptic Nonlocal Equations Set in Cylinders. To appear in Asymptotic Analysis.

[47] K. Yeressian, Spatial asymptotic behaviour of elliptic equations and variational inequalities. Thesis University of Zürich, (2010). 